\title{
Periodontal pockets: a potential reservoir for SARS-CoV-2?
}

\author{
Zahi Badran 1,2, Alexis Gaudin ${ }^{3}$, Xavier Struillou ${ }^{1}$, Gilles Amador ${ }^{4}$, Assem Soueidan ${ }^{1 *}$ \\ ${ }^{1}$ Department of Periodontology (Rmes U1229, UIC 11), Faculty of Dental Surgery, University of Nantes (CHU de Nantes), Nantes, France \\ ${ }^{2}$ Faculty of Dentistry, McGill University, Montreal, Canada \\ ${ }^{3}$ Department of Endodontics (Rmes U1229, UIC 11), Faculty of Dental Surgery, University of Nantes (CHU de Nantes), Nantes, France \\ ${ }^{4}$ Department of Dental public health (UPRES EA 3826, UIC 11), Faculty of Dental Surgery, University of Nantes (CHU de Nantes), Nantes, \\ France
}

* Corresponding author: Prof Assem Soueidan, Faculty of Dental Surgery, 1 place A. Ricordeau, 44042, Nantes, France, email: assem.soueidan@univ-nantes.fr

\begin{abstract}
Periodontal pockets are the major clinical manifestation of Periodontitis, a chronic inflammatory oral disease affecting the teeth-supporting tissues and has high prevalence in the adult population. Periodontal pockets are ideal environments for subgingival bacterial biofilms, that interact with the supragingival oral cavity, mucosal tissues of the pocket and a peripheral circulatory system. Periodontal pockets have been found to harbor viral species such as the Herpes simplex viruses' family. Recently, the SARS-CoV-2 has gained major interest of the scientific/medical community as it caused a global pandemic (Covid-19) and paralyzed the globe with high figures of infected people worldwide. This virus behavior is still partially understood, and by analyzing some of its features we hypothesized that periodontal pocket could be a favorable anatomical niche for the virus and thus acting as a reservoir for SARS-CoV-2.
\end{abstract}




\section{Introduction}

Periodontal disease are highly prevalent [1] inflammatory chronic diseases, initiated by bacterial infection and leading to the destruction of teeth-supporting tissues [2] and hence the shift from a healthy gingival sulcus to the formation of periodontal pockets (PP).This is done after a pathological apical migration of the supracrestal attachment tissues, coupled with alveolar bone loss. PP have specific subgingival environments with a dental root wall permitting the development of complex subgingival biofilms and a mucosal wall formed by an ulcerated epithelium with an exposed connective tissue and its vascular ramifications [3].

SARS-CoV-2 is a newly discovered virus, from the Coronavirus family. Its recent outbreak caused a major pandemic of the coronavirus disease (Covid-19). The primary entry of this virus is believed to be by projected droplets leading to a first contact and colonization of cells in the oral cavity, nose or eyes [4]. People diagnosed positive for this virus will develop variable symptoms in mild cases (fever, cough, headache, anosmia, ageusia etc.). In moderate to severe cases, respiratory failure will occur and could in some cases lead to intensive care unit hospitalization and eventually death [5]. The believed target cells of this newly discovered virus are within the respiratory tract but other locations such as the heart or the gastro-intestinal tract are plausible targets [6]. Until now, mainly nasal and sometimes oropharyngeal swabs, has been used to determine if patients are Covid-positive or negative, by using a polymerase chain reaction test (PCR) [7]. Nevertheless, cases of Covid-negative patients by PCR test , showing obvious clinical signs of the disease have been reported [8, 9]. Also, evidence of patients showing recurrence of clinical signs after recovery are emerging [10]. Retrospective data has also shown the possibility of SARS-CoV-2 reactivation [11]. All these observations have risen many questions on the accuracy of the tests, existence of different virus strains, possibility of reinfection etc. One specific hypothetical question would be if anatomical niches exist in the body, other than the initial nasopharyngeal/oropharyngeal tracts, where samplings are performed. These hypothetical niches could act as reservoirs for SARS-CoV-2, whether in an active or latent state. 


\section{The hypothesis}

PP could be a niche for SARS-CoV-2 virus infection. The virus could find in PP a favorable environment to replicate and to reach continuously the oral cavity and mix with saliva, or to migrate systemically using the capillary periodontal complex. Hence, PP are plausible reservoirs for Sars-Cov-2 viruses.

\section{Viral presence in periodontal pockets}

The viral detection in PP, has been established in numerous publications [12-19]. Regarding the dental literature, the most frequently detected viruses in PP, other than bacteriophages in dental plaque [20], are Herpes simplex virus (HSV), Epstein-Barr virus (EBV) and Human Cytomegalovirus (HCMV) [15]. Median prevalence figures in PP of different forms of periodontitis are $26-78 \%, 46-58 \%$ and $42-$ $58 \%$ for HSV, EBV and HCMV respectively [21]. Possible sources of initial viral infection of periodontal tissues infection could be as follow: a direct infection of gingival epithelial cells exposed to the oral cavity, virus migration through the blood stream or infected immune cells in the periodontal inflammatory infiltrate [22]. These previous observations point to the fact that PP are in compatible environments for viral infection and survival. Furthermore, with continuous inflammatory response associated to periodontal disease, immune cells potentially infected with viruses could reach the PP connective tissue and migrate to the subgingival area. Viral presence detected with conventional methods, mainly PCR, was found in many localization of PP: gingival tissues [17], subgingival plaque [23] and in gingival crevicular fluid (GCF) [24]. The latter is an inflammatory exudate generated in periodontal tissues and released in the PP [25] to "flush" the subgingival space . Also, GCF composition is rich with epithelial and immune cells, antibodies, microbial metabolites, biomarkers etc. If GCF could harbor SARS-CoV-2 released from infected periodontal cells or by terminal capillary complexes in periodontal tissues, viruses could attain oral cavity by the PP entry and hence, mix with saliva that was found to contains detectable SARS-CoV-2 [26]. The presence of SARS-CoV-2 in GCF would be synchronous with the viremia, occurring after an initial colonization of target cells.

\section{Oral expression of SARS-CoV-2 receptors}


Angiotensin converting enzyme-2 (ACE-2) has been considered the main receptor for the virus entry into target cells [27]. Furin, a proprotein convertase is implicated in virus infection by cleaving viral envelope glycoproteins [28]. ACE-2 has been found to be expressed by various types of cells such as pulmonary cells, nasopharyngeal cells, salivary gland cells etc. In recent published preprint data, Cells from the oral cavity have been found to highly express ACE-2, in a comparable manner to lung cells [29]. Furthermore, besides ACE-2, Furin is also expressed in oral epithelial cells [30]. Previous published data has also confirmed the expression of ACE- 2 in gingival and periodontal ligament fibroblasts [31] in rat and human tissues.

Recently, preliminary data suggested a novel infection route by SARS-CoV-2. The latter could infect cells by using its spike protein to bind to the Cluster of Differentiation 147 (CD 147) [32] on cell membranes and not necessarily ACE-2. If this was to be confirmed in future studies, cells expressing CD 147 would be also considered at high risk of infection by SARS-CoV-2. Previous data has shown that oral epithelial cells, that constitute the buccal and subgingival component of PP cells, express CD 147 [33]. Furthermore, gingival epithelium expression of CD 147 is increased in cells harvested from periodontitis patients [34].

\section{Conclusion}

PP are peculiar isolated environments, presenting proper biological dynamics, with two-way interactions with the oral cavity on one hand and the systemic circulatory system via gingival peripheral blood vessels one the other hand. Furthermore, if bacterial biofilms in PP has been the main focus of the scientific dental community, viral presence and its implication in periodontal health and disease has become progressively plausible over the time with the acquired cumulative knowledge [35]. It is becoming more widely accepted that besides the bacterial challenge, viruses from PP could infect distant organs and hence generate focal infections [36]. Virus from PP would join oral cavity via GCF and the mixing with saliva when leaving the subgingival area. Another potential viral migration track would be the passage to the periodontal capillary system and then to the systemic blood stream. 
In the case of SARS-CoV-2, known and suspected features of this virus' affinity to specific membrane receptors, are compatible with a hypothesized affinity to PP cells. This could involve the outer/interior epithelial lining or the gingival/periodontal ligament fibroblasts.

On the other hand, it has been shown that periodontium-associated viruses can infect immune cells like macrophages, $\mathrm{T}$ lymphocytes etc. [37]. These are continuously arriving to the inflammatory infiltrate in the mucosal wall of PP. This could be another potential source of viruses found in PP. The question of whether SARS-CoV-2 could infect these specific types of inflammatory cells is open to debate. Previous data related to SARS-CoV indicated that the latter can infect et replicate in mononuclear cells but for a limited time [38]. A similar pattern of actions has been described for SARSCoV-2 infecting T-Lymphocytes [39], but with an apoptosis trigger of the infected cells. It is still unclear if the SARS-CoV-2 replication is deficient in all inflammatory cells. Also, the infection of endothelial cells by SARS-CoV-2 seems possible [40].

Future studies should elucidate the various patterns of SARS-CoV-2 infection-replication-migration, in relation to each specific target cell families and to the plausibility of reactivation of the virus in already healed patients [11]. In view of the existing limited data, the hypotheses that PP could act as a reservoir of SARS-CoV-2 relays on consistent and plausible observations. In our clinical research unit (Nantes University Hospital, France), we have an ongoing project to collect GCF/subgingival plaque in PP from Covid-positive patients in order to assess the proposed hypothesis.

If this is to be confirmed, future directions would be to use PP GCF/plaque sampling as a testing tool for Covid-19. Furthermore, periodontal therapy would be considered a parameter of care in the global clinical management of Covid-positive patients.

\section{Conflict of interest}

Authors declare no conflict of interest 


\section{Acknowledgments}

We would like to thank Mrs M. Cheraud-Carpentier, Mr A. Legrand and $\operatorname{Dr}$ O. Bandiaky for their valuable contribution to the "Cov-oral" research project, inspired by the hypotheses described in this manuscript

\section{References}

[1] Helmi MF, Huang H, Goodson JM, Hasturk H, Tavares M, Natto ZS. Prevalence of periodontitis and alveolar bone loss in a patient population at Harvard School of Dental Medicine. BMC Oral Health. 2019;19:254.

[2] Shi M, Wei Y, Hu W, Nie Y, Wu X, Lu R. The Subgingival Microbiome of Periodontal Pockets With Different Probing Depths in Chronic and Aggressive Periodontitis: A Pilot Study. Front Cell Infect Microbiol. 2018;8:124.

[3] Takata T, Donath K. The mechanism of pocket formation. A light microscopic study on undecalcified human material. J Periodontol. 1988;59:215-21.

[4] Lo Giudice R. The Severe Acute Respiratory Syndrome Coronavirus-2 (SARS CoV-2) in Dentistry. Management of Biological Risk in Dental Practice. Int J Environ Res Public Health. 2020;17.

[5] Lupia T, Scabini S, Mornese Pinna S, Di Perri G, De Rosa FG, Corcione S. 2019 novel coronavirus (2019-nCoV) outbreak: A new challenge. J Glob Antimicrob Resist. 2020;21:22-7.

[6] Astuti I, Ysrafil. Severe Acute Respiratory Syndrome Coronavirus 2 (SARS-CoV-2): An overview of viral structure and host response. Diabetes Metab Syndr. 2020;14:407-12.

[7] Petruzzi G, De Virgilio A, Pichi B, Mazzola F, Zocchi J, Mercante G, et al. COVID-19: Nasal and oropharyngeal swab. Head Neck. 2020.

[8] Bullis SSM, Crothers JW, Wayne S, Hale AJ. A Cautionary Tale of False-Negative Nasopharyngeal COVID-19 Testing. IDCases. 2020:e00791. 
[9] Wang W, Xu Y, Gao R, Lu R, Han K, Wu G, et al. Detection of SARS-CoV-2 in Different Types of Clinical Specimens. JAMA. 2020.

[10] Lan L, Xu D, Ye G, Xia C, Wang S, Li Y, et al. Positive RT-PCR Test Results in Patients Recovered From COVID-19. JAMA. 2020;323:1502-3.

[11] Ye G, Pan Z, Pan Y, Deng Q, Chen L, Li J, et al. Clinical characteristics of severe acute respiratory syndrome coronavirus 2 reactivation. J Infect. 2020;80:e14-e7.

[12] Pallos D, Ruivo GF, Ferrari-Junior SH, Pannuti CS, Perozini C, Sarmento DJS, et al. Periodontal disease and detection of human herpesviruses in saliva and gingival crevicular fluid of chronic kidney disease patients. J Periodontol. 2020.

[13] Shah R, Mehta DS. Prevalence of herpesviruses in gingivitis and chronic periodontitis: relationship to clinical parameters and effect of treatment. J Indian Soc Periodontol. 2016;20:279-85.

[14] Dawson DR, 3rd, Wang C, Danaher RJ, Lin Y, Kryscio RJ, Jacob RJ, et al. Salivary levels of EpsteinBarr virus DNA correlate with subgingival levels, not severity of periodontitis. Oral Dis. 2009;15:554-9. [15] Cappuyns I, Gugerli P, Mombelli A. Viruses in periodontal disease - a review. Oral Dis. 2005;11:21929.

[16] Pereira CM, Gasparetto PF, Correa ME, Costa FF, de Almeida OP, Barjas-Castro ML. Human herpesvirus 6 in oral fluids from healthy individuals. Arch Oral Biol. 2004;49:1043-6.

[17] Contreras A, Nowzari H, Slots J. Herpesviruses in periodontal pocket and gingival tissue specimens. Oral Microbiol Immunol. 2000;15:15-8.

[18] Contreras A, Slots J. Active cytomegalovirus infection in human periodontitis. Oral Microbiol Immunol. 1998;13:225-30.

[19] Parra B, Slots J. Detection of human viruses in periodontal pockets using polymerase chain reaction. Oral Microbiol Immunol. 1996;11:289-93.

[20] Edlund A, Santiago-Rodriguez TM, Boehm TK, Pride DT. Bacteriophage and their potential roles in the human oral cavity. J Oral Microbiol. 2015;7:27423. 
[21] Aggarwal T, Lamba AK, Faraz F, Tandon S. Viruses: Bystanders of periodontal disease. Microb Pathog. 2017;102:54-8.

[22] Miller CS. Viruses: are they really culprits for periodontal disease? A critical review? J Investig Clin Dent. 2014;5:243.

[23] Das S, Krithiga GS, Gopalakrishnan S. Detection of human herpes viruses in patients with chronic and aggressive periodontitis and relationship between viruses and clinical parameters. J Oral Maxillofac Pathol. 2012;16:203-9.

[24] Grenier G, Gagnon G, Grenier D. Detection of herpetic viruses in gingival crevicular fluid of patients suffering from periodontal diseases: prevalence and effect of treatment. Oral Microbiol Immunol. 2009;24:506-9.

[25] Subbarao KC, Nattuthurai GS, Sundararajan SK, Sujith I, Joseph J, Syedshah YP. Gingival Crevicular Fluid: An Overview. J Pharm Bioallied Sci. 2019;11:S135-S9.

[26] To KK, Tsang OT, Chik-Yan Yip C, Chan KH, Wu TC, Chan JMC, et al. Consistent detection of 2019 novel coronavirus in saliva. Clin Infect Dis. 2020.

[27] Zou X, Chen K, Zou J, Han P, Hao J, Han Z. Single-cell RNA-seq data analysis on the receptor ACE2 expression reveals the potential risk of different human organs vulnerable to 2019-nCoV infection. Front Med. 2020;14:185-92.

[28] Izaguirre G. The Proteolytic Regulation of Virus Cell Entry by Furin and Other Proprotein Convertases. Viruses. 2019;11.

[29] Chao Wu MZ. Single-cell RNA expression profiling shows that ACE2, the putative receptor of COVID-2019, has significant expression in nasal and mouth tissue, and is co-expressed with TMPRSS2 and not co-expressed with SLC6A19 in the tissues. PREPRINT (Version 1) available at Research Square. 12 MARCH 2020.

[30] Lin B-p, Zhong M, Gao H-b, Wu K-b, Liu M-x, Liu C, et al. Significant expression of FURIN and ACE2 on oral epithelial cells may facilitate the efficiency of 2019-nCov entry. bioRxiv. 2020:2020.04.18.047951. 
[31] Santos CF, Morandini AC, Dionisio TJ, Faria FA, Lima MC, Figueiredo CM, et al. Functional Local Renin-Angiotensin System in Human and Rat Periodontal Tissue. PLoS One. 2015;10:e0134601.

[32] Wang K, Chen W, Zhou Y-S, Lian J-Q, Zhang Z, Du P, et al. SARS-CoV-2 invades host cells via a novel route: CD147-spike protein. bioRxiv. 2020:2020.03.14.988345.

[33] Feldman M, La VD, Lombardo Bedran TB, Palomari Spolidorio DM, Grenier D. Porphyromonas gingivalis-mediated shedding of extracellular matrix metalloproteinase inducer (EMMPRIN) by oral epithelial cells: a potential role in inflammatory periodontal disease. Microbes Infect. 2011;13:1261-9. [34] Wang J, Yang D, Li C, Shang S, Xiang J. Expression of extracellular matrix metalloproteinase inducer glycosylation and caveolin-1 in healthy and inflamed human gingiva. J Periodontal Res. 2014;49:197204.

[35] Chen C, Feng P, Slots J. Herpesvirus-bacteria synergistic interaction in periodontitis. Periodontol 2000. 2020;82:42-64.

[36] Slots J. Focal infection of periodontal origin. Periodontol 2000. 2019;79:233-5.

[37] Contreras A, Zadeh HH, Nowzari H, Slots J. Herpesvirus infection of inflammatory cells in human periodontitis. Oral Microbiol Immunol. 1999;14:206-12.

[38] Li L, Wo J, Shao J, Zhu H, Wu N, Li M, et al. SARS-coronavirus replicates in mononuclear cells of peripheral blood (PBMCs) from SARS patients. J Clin Virol. 2003;28:239-44.

[39] Wang X, Xu W, Hu G, Xia S, Sun Z, Liu Z, et al. SARS-CoV-2 infects T lymphocytes through its spike protein-mediated membrane fusion. Cellular \& Molecular Immunology. 2020.

[40] Varga Z, Flammer AJ, Steiger P, Haberecker M, Andermatt R, Zinkernagel AS, et al. Endothelial cell infection and endotheliitis in COVID-19. Lancet. 2020;395:1417-8. 